\title{
Inactivation of Pseudomonas aeruginosa biofilm after ultraviolet light-emitting diode treatment: a comparative study between ultraviolet $C$ and ultraviolet $B$
}

\author{
Aikaterini Argyraki \\ Merete Markvart \\ Lars Bjørndal \\ Thomas Bjarnsholt \\ Paul Michael Petersen
}




\title{
Inactivation of Pseudomonas aeruginosa biofilm after ultraviolet light-emitting diode treatment: a comparative study between ultraviolet C and ultraviolet B
}

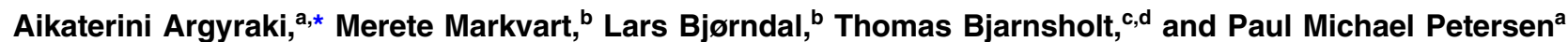 \\ aTechnical University of Denmark, Department of Photonics Engineering, Roskilde, Denmark \\ bUniversity of Copenhagen, Cariology and Endodontics, Department of Odontology, Faculty of Health and Medical Science, \\ Copenhagen N, Denmark \\ cUniversity of Copenhagen, Costerton Biofilm Center, Department of Immunology and Microbiology, Faculty of Health and \\ Medical Science, Copenhagen N, Denmark \\ ${ }^{\mathrm{d}}$ Rigshospitalet, Department of Clinical Microbiology, Copenhagen Ø, Denmark
}

\begin{abstract}
The objective of this study was to test the inactivation efficiency of two different light-based treatments, namely ultraviolet B (UVB) and ultraviolet C (UVC) irradiation, on Pseudomonas aeruginosa biofilms at different growth stages $(24,48$, and $72 \mathrm{~h}$ grown). In our experiments, a type of AIGaN light-emitting diodes (LEDs) was used to deliver UV irradiation on the biofilms. The effectiveness of the UVB at $296 \mathrm{~nm}$ and UVC at $266 \mathrm{~nm}$ irradiations was quantified by counting colony-forming units. The survival of less mature biofilms (24 $\mathrm{h}$ grown) was studied as a function of UV-radiant exposure. All treatments were performed on three different biological replicates to test reproducibility. It was shown that UVB irradiation was significantly more effective than UVC irradiation in inactivating $P$. aeruginosa biofilms. UVC irradiation induced insignificant inactivation on mature biofilms. The fact that the UVB at $296 \mathrm{~nm}$ exists in daylight and has such disinfection ability on biofilms provides perspectives for the treatment of infectious diseases. $\odot$ The Authors. Published by SPIE under a Creative Commons Attribution 3.0 Unported License. Distribution or reproduction of this work in whole or in part requires full attribution of the original publication, including its DOI. [DOI: $10.1117 / 1 . J B O .22 .6 .065004]$
\end{abstract}

Keywords: disinfection; ultraviolet light; ultraviolet C; ultraviolet B; light-emitting diodes; Pseudomonas aeruginosa.

Paper 170102PR received Feb. 13, 2017; accepted for publication May 30, 2017; published online Jun. 24, 2017.

\section{Introduction}

Biofilm-contaminated medical devices are believed to be a common cause for hospital-acquired infections. ${ }^{1}$ Moreover, biofilms are implicated in chronic infections, such as chronic wounds and tissue filler-, implant-, and catheter-associated infections. ${ }^{2}$ Traditionally microbial infections are treated by antibiotics that either kill the microbe or inhibit the contaminated area from spreading. ${ }^{3}$ However, the problem with this approach is that antibiotic resistance is developed. ${ }^{4}$ Furthermore, biofilms exhibit greater tolerance to antibiotics and antimicrobial stressors than planktonic organisms of the same species. ${ }^{5}$ As a consequence, researchers have turned their interest toward alternative disinfection approaches, including bacteriophages, ${ }^{6}$ bacteriocins, ${ }^{7}$ and light-based treatments. ${ }^{8} \mathrm{UV}$ irradiation, ${ }^{9,10}$ photodynamic therapy, ${ }^{11,12}$ blue,${ }^{13,14}$ and near-infrared light ${ }^{15,16}$ are all noninvasive methods and have been reported to have the ability to inactivate microorganisms. Moreover, the expectation that the bacteria will develop light-resistant genes is low. ${ }^{8}$ On the other hand, light-based solutions demand effective light delivery. Thus, the potential applications are confined by penetration depth of light to the region of interest. For this reason, branches of medicine, such as dermatology and dentistry were the first to adopt the technology due to easier optical access to the region of interest.

*Address all correspondence to: Aikaterini Argyraki, E-mail: aikar@fotonik.dtu .dk
In this work, the efficiencies of two different light-based disinfection treatments were tested, namely ultraviolet B (UVB) and ultraviolet $\mathrm{C}$ (UVC) irradiation, on $P$. aeruginosa biofilms at different growth stages. Their antibacterial action was studied as a function of radiant exposure. The survival curves were modeled using GInaFiT ${ }^{17}$ (Geeraerd and Van Impe Inactivation Model Fitting Tool).

The disinfection ability of UV light-emitting diodes (LEDs) could offer new possibilities for potential disinfection applications not only in terms of cleaning instruments, equipment, and clinical surfaces but also in terms of potential treatments.

\section{Materials and Methods}

\subsection{Ultraviolet Treatments}

The UV LEDs that were used to deliver the irradiation to the biofilms were purchased from Sensor Electronic Technology, Inc. (SETi, Columbia, South Carolina; TO3 package, hemispherical lens window). The diodes were operated in constant current mode. The spectral power distribution of the LEDs is shown in Fig. 1. The setup for performing the irradiations is shown in Fig. 2. The irradiation of the UV-LEDs was measured by an external optical probe (EOP-146, Instrument Systems $\mathrm{GmbH}$, Munich, Germany) and a monochromator. The spectrometer coupled to the monochromator was a SPECTRO 320 (D) Release 5 (Instrument Systems GmbH). Details about the irradiation measurements protocol can be found in Barnkob 


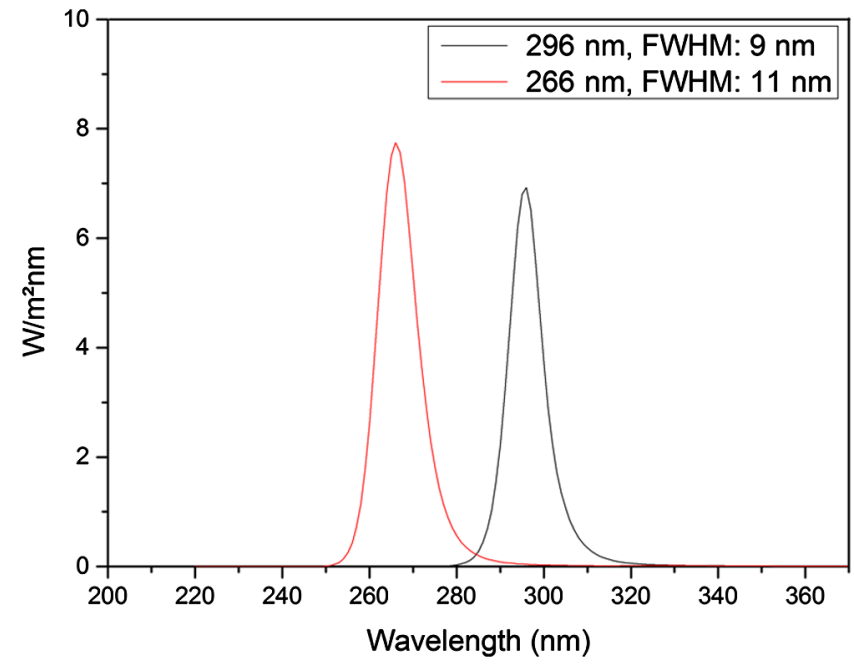

Fig. 1 Spectral irradiance of the UVB (black curve) and UVC LEDs (red curve) used for the irradiation of the biofilms.

et al. ${ }^{18}$ The irradiance delivered on the biofilms was also cross confirmed with a portable radiometer (NIST-certified UV radiometer); the distances the irradiance measurements were taken $(1.5 \pm 0.1 \mathrm{~cm})$ were the same distances that the exposures were conducted.

In all exposures, the distance between the LED and the biofilm (circular entity with diameter $1.0 \pm 0.2 \mathrm{~cm}$ ) was kept constant at $1.5 \pm 0.2 \mathrm{~cm}$ (the error originates from the agar height in the Petri dish). All biofilms, before and after treatments, were stored in a UV-free environment. As a standard, all treatments were performed on three different biological replicates to test the reproducibility.

\subsection{Biofilm Preparation}

A cellulose nitrate membrane filter (pore size: $0.2-\mu \mathrm{m}$ diameter: $25 \mathrm{~mm}$, Whatman $\mathrm{GmbH}$, Germany) was placed directly on the agar plate. The plate and filter were dried for $30 \mathrm{~min}$ at $37^{\circ} \mathrm{C}$ before the bacterial overnight $(\mathrm{ON})$ culture was added in a single spot (20- $\mu \mathrm{L}$ bacterial suspension) on the membrane filter. The bacteria were spot inoculated onto the filter directly from the ON culture. The $P$. aeruginosa used for the experiments was obtained from the Pseudomonas Genetic Stock Center (strain $\left.\mathrm{PAO} 1^{19}\right)$. The biofilms were grown on AB-trace glucose $(0.5 \%)$ (ABTG) plates and incubated for 24,48 , or $72 \mathrm{~h}$ at $37^{\circ} \mathrm{C}$. A more detailed description for the $\mathrm{ON}$ cultures and filter biofilms methodology ("the micropore assay") can be found in Bjarnsholt et al. ${ }^{20}$ For a mature biofilm (either grown for 48 or $72 \mathrm{~h}$ ) to develop, the membrane filter containing the growing biofilm was transferred to a fresh, dried ABTG plate every $24 \mathrm{~h}$. In this way, the fresh media available to the biofilm facilitated growth.

\subsection{Colony-Forming Unit Determination}

Filters with the biofilms growing on top were transferred with sterile forceps to a $15 \mathrm{~mL}$ Falcon tube containing $5 \mathrm{~mL}$ of saline $(0.9 \% \mathrm{NaCl})$. Samples were mixed for $15 \mathrm{~s}$, degassed for $5 \mathrm{~min}$, and sonicated for $5 \mathrm{~min}$ in an ultrasonication bath to release the bacteria from the filter. Sonication fluid was serial diluted from $10^{-1}$ to $10^{-8}$ and $20 \mu \mathrm{L}$ of all dilutions were spotted once on LB agar plates followed by $\mathrm{ON}$ incubation at $37^{\circ} \mathrm{C}$. The colonyforming units (CFUs) were counted after $24 \mathrm{~h}$ of incubation in the dark at $37^{\circ} \mathrm{C}$. Note that CFUs are per initial volume and were calculated with the weighted average method. ${ }^{21}$ An example of how the CFUs looked after counting is shown in Fig. 3. Control samples, i.e., biofilms that were not exposed to UV irradiation, were plated every hour and included in the study as a reference for growth.

\subsection{Experimental Design}

The objective was to determine how the selected UV region, namely UVB versus UVC, and radiant exposure will influence the viability of $P$. aeruginosa biofilms. The radiant exposure for

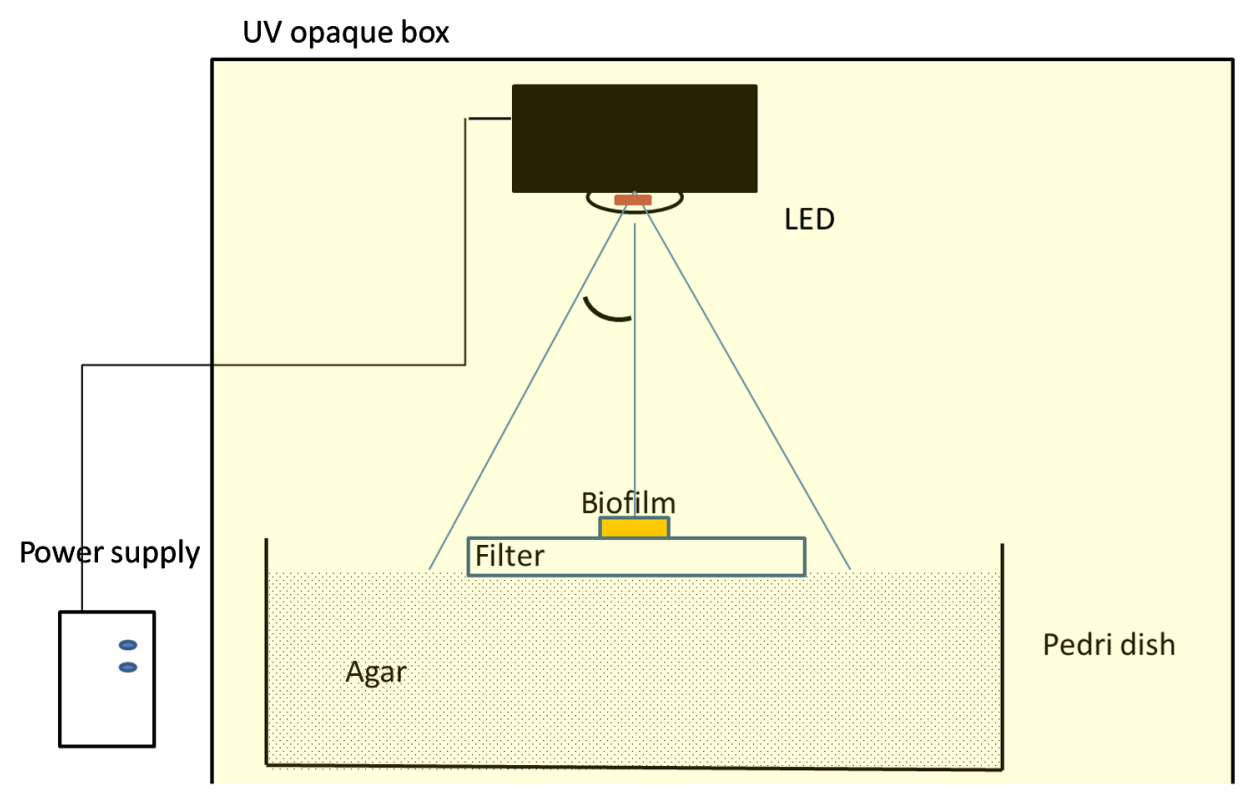

Fig. 2 Schematic sketch of the UV irradiation setup. The agar plate carrying the biofilm is illuminated by the UV LED in a UV-opaque box. 


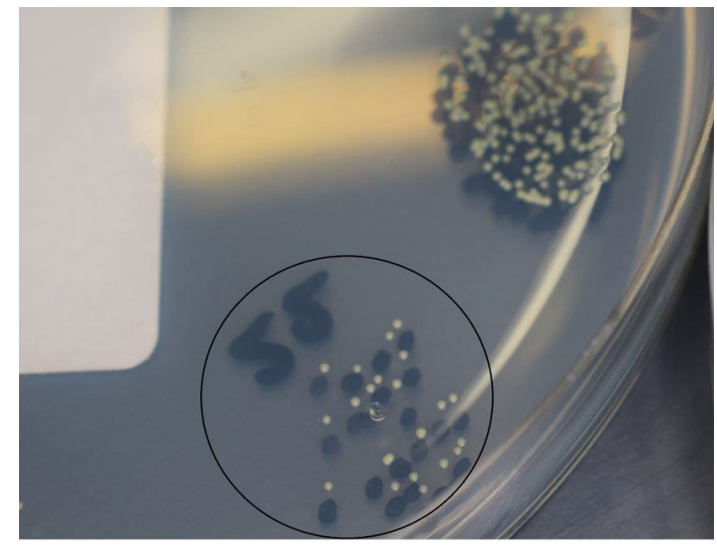

Fig. 3 Typical picture of a labeled agar plate after regrowth (control sample). At higher dilutions, countable and well-separated CFUs are formed, as indicated by the black circle. The yellowish spots are the bacterial colonies, the back spots are the marks made by the technician (at the other side of the plate) while counting. The next aggregation of colonies (move counterclockwise) is from one order lower dilution; and the colonies (yellowish spots) are occasionally overlapping.

less mature biofilms was investigated in the range (approximately) from 70 to $10,000 \mathrm{~J} / \mathrm{m}^{2}$. The irradiance was varied between $18.6 \pm 0.2 \mathrm{~W} / \mathrm{m}^{2}$ and $108 \pm 11 \mathrm{~W} / \mathrm{m}^{2}$ with the UVC diode. For the UVB diode, the irradiance was varied between $1 \pm 0.1 \mathrm{~W} / \mathrm{m}^{2}$ and $14.8 \pm 0.2 \mathrm{~W} / \mathrm{m}^{2}$. In all treatments, the exposure time was $<12 \mathrm{~min}$.

Mature biofilms (48 and $72 \mathrm{~h}$ grown) were treated with radiant exposure around $20,000 \mathrm{~J} / \mathrm{m}^{2}$, and effectiveness of the treatments (UVB and UVC) was compared to control (no treatment) and less mature biofilms ( $24 \mathrm{~h}$ grown). For testing the effect of maturity on the effectiveness of the treatments, the exposures were performed on three independent biological replicates and executed twice (two technical replicates). Pairwise $t$-tests were used to detect inactivation efficiency differences among treatments $\left(20,000 \mathrm{~J} / \mathrm{m}^{2}\right)$ for the different growth stages.

\subsection{Modeling of Biofilm Survival}

GInaFiT $^{17}$ a freeware add-in for Microsoft ${ }^{\circledR}$ Excel, was used to model the biofilm survival curves. Modeling of bacterial survival can provide some insight into the mechanisms behind inactivation and provide guidelines for prediction of required doses to succeed a specific level of inactivation. Microbial inactivation has traditionally been described by first-order kinetics. The GInaFiT tool supports testing of nine types of microbial survival models, and five statistical measures (i.e., sum of squared errors, mean sum of squared errors and its root, $R^{2}$, and adjusted $R^{2}$ ) are provided to monitor the best fit ( $f$ function). Here, a choice of five suitable models ${ }^{22-26}$ was applied to the mean values (from three technical replicates) of log survival obtained from one biological replicate (replicate 3 )

$$
\begin{aligned}
\log \text { survival } & =\log \frac{N_{\text {treated }}}{N_{\text {control }}}=-\left(\log N_{\text {control }}-\log N_{\text {treated }}\right) \\
& =-\log \text { inactivation }=f(\text { dose })
\end{aligned}
$$

where $N_{\text {treated }}$ is the number of CFUs after a UV-radiant exposure $\left(\mathrm{J} / \mathrm{m}^{2}\right)$ is delivered to the biofilm and $N_{\text {control }}$ is the number

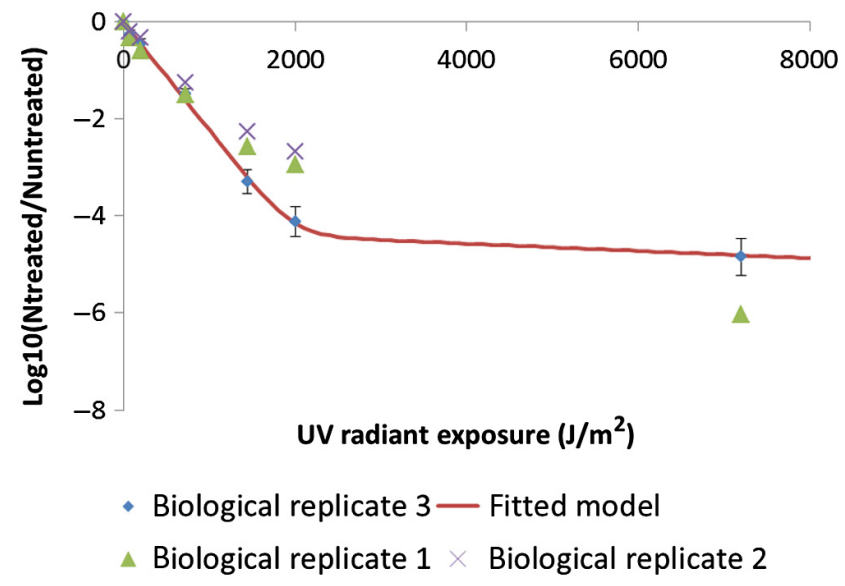

Fig. 4 UVB-radiant exposure-dependent antibacterial action on $P$. aeruginosa biofilms. "Biphasic model" fit to the experimental data. This model requires a minimal 10 points to ensure validity. The error bars for biological replicate 3 represent propagation of uncertainty for the function of log survival, originating from three technical replicates.

of CFUs on the controls (not UV exposed). When zero counts are observed in $N_{\text {treated }}$, the log survival is not calculated.

\section{Results}

\subsection{Ultraviolet B Inactivation Rate (Less Mature Biofilms)}

The log survival values of $P$. aeruginosa biofilms, after being exposed to UVB irradiation with a central wavelength at $296 \mathrm{~nm}$, as a function of UV-radiant exposure (dose) are presented in Fig. 4. Most models demonstrated a good fit to the experimental data $\left(R^{2} \geq 0.9\right)$, except the linear model. ${ }^{22}$ The "biphasic" model ${ }^{26}$ fit best to the data (Fig. 4). However, this model requests minimal 10 points to ensure validity. The statistical measures of the models applied are presented in Table 1. If we use the concept of reliability engineering, the reliable dose $d_{R}{ }^{27}$ (dose needed to reduce number of microorganisms by a factor of 10) is calculated to be $638 \mathrm{~J} / \mathrm{m}^{2}$ (from the "Weibull model," the hazard rate was found to be $\alpha=54.86$ and the shape parameter $\beta=0.34$ ).

The GInaFiT tool predicts that the required dose for achieving a 4-log reduction in the CFUs is $1900 \mathrm{~J} / \mathrm{m}^{2}$, in accordance with the experimentally observed value (at $2000 \mathrm{~J} / \mathrm{m}^{2}, 4.1-\mathrm{log}$ reduction). No CFU was observed when a dose of $10,000 \mathrm{~J} / \mathrm{m}^{2}$ was delivered to the biofilm (independently of biological replicate), indicating total inactivation; potential presence of bacteria could not be detected since the treatment challenged the detection limit of the method used (drop plate technique ${ }^{28}$ ), nor were CFUs observed for biological replicate two, already at a radiant exposure of $7200 \mathrm{~J} / \mathrm{m}^{2}$.

\subsection{Ultraviolet C Inactivation Rate (Less Mature Biofilms)}

The antibacterial action of UVC irradiation, with central wavelength at $266 \mathrm{~nm}$ is shown in Fig. 5 with a "Weibull model fit. ${ }^{24 \text { ", }}$ With $\alpha=9870$ and $\beta=0.44$, the reliable dose $d_{R}{ }^{27}$ was calculated to be $65,722 \mathrm{~J} / \mathrm{m}^{2}$. The statistical measures of the models applied are shown in Table 1. The GInaFiT tool in this case was 
Table 1 Statistical measures of the models applied to the experimental data for log reduction of $P$. aeruginosa by UVB (and UVC). In cases where modeling was not applicable, the indication NA is registered.

\begin{tabular}{|c|c|c|c|c|c|c|}
\hline Fitted model & MSE & RMSE & $R^{2}$ & Adjusted $R^{2}$ & SSE & D $4 \log$ \\
\hline Log-linear & $1.6(0.03)$ & $1.28(0.16)$ & $0.69(0.84)$ & $0.63(0.80)$ & $9.84(0.13)$ & 8700 (NA) \\
\hline Weibull & $0.60(0.01)$ & $0.78(0.1)$ & $0.90(0.95)$ & $0.87(0.92)$ & $2.99(0.04)$ & 3300 (NA) \\
\hline Biphasic & $0.01(0.01)$ & $0.09(0.08)$ & $0.9989(0.98)$ & $0.9980(0.95)$ & $0.04(0.02)$ & 1900 (NA) \\
\hline $\log +$ tail & $0.014(0.02)$ & $0.12(0.12)$ & $0.9978(0.92)$ & $0.9970(0.88)$ & $0.07(0.06)$ & $1900(\mathrm{NA})$ \\
\hline Albert (Weibull and tail) & 0.017 (NA) & 0.13 (NA) & 0.9978 (NA) & 0.9960 (NA) & 0.07 (NA) & 1900 (NA) \\
\hline
\end{tabular}

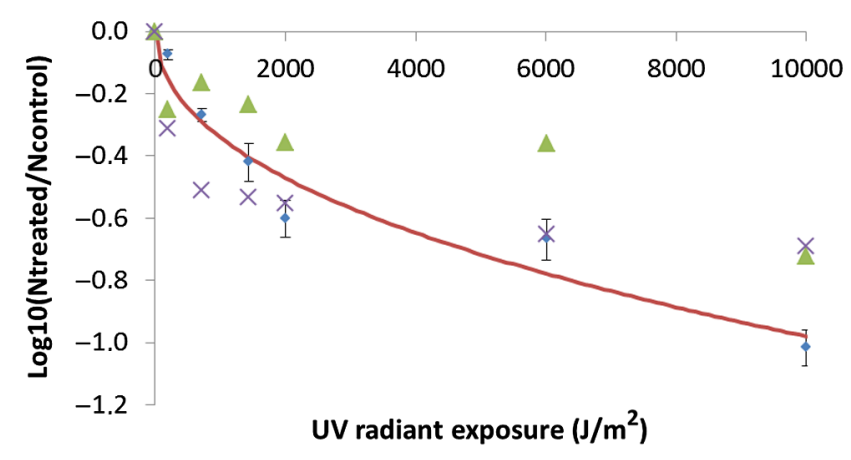

- Biological replicate 3 - Fitted model

$\triangle$ Biological replicate $2 \times$ Biological replicate 3

Fig. 5 UVC-radiant exposure-dependent antibacterial action on $P$. aeruginosa biofilms (experimental data and Weibull fit). The error bars for biological replicate 3 represent propagation of uncertainty for the function of log survival, originating from three technical replicates.

unable to predict the required dose for achieving a 4-log reduction (maximum log reduction achieved experimentally was 1).

\subsection{Mature Biofilms}

The effect of the growth stage of the biofilm to the observed efficacy of the applied treatments is summarized in Fig. 6. No inactivation effect was observed with UVC (radiant exposure around $20,000 \mathrm{~J} / \mathrm{m}^{2}$ ) on $48 \mathrm{~h}$ mature biofilms; CFU counts were similar to the nontreated samples. Therefore, the treatment was not attempted on biofilms grown for $72 \mathrm{~h}$. Only a modest inactivation was achieved on the less mature biofilms around 1$\log$ reduction $(0.8 \pm 0.3 \log$ reductions $)$. To the contrary, UVB (radiant exposure around $20,000 \mathrm{~J} / \mathrm{m}^{2}$ ) was able to inactivate effectively mature biofilms grown for $48 \mathrm{~h}(3.2 \pm 1.3 \mathrm{log}$ reduction) and for $72 \mathrm{~h}(2.9 \pm 0.7 \mathrm{log}$ reduction). However, the efficiency of inactivation decreased with maturity from zero counts for less mature biofilms to around 3-log reduction for mature biofilms. It is interesting to observe that the log reduction achieved by the UVB treatment varies when the biofilms are mature but are "straightforward" for the less mature biofilms. According to the pairwise $t$-tests, there was a significant increase in counts for the control from less mature to mature biofilms $(p=0.003)$. UVB treatment was significantly more effective than UVC, both for biofilms grown for 24 and $48 \mathrm{~h}$ ( $p \leq 0.004)$.

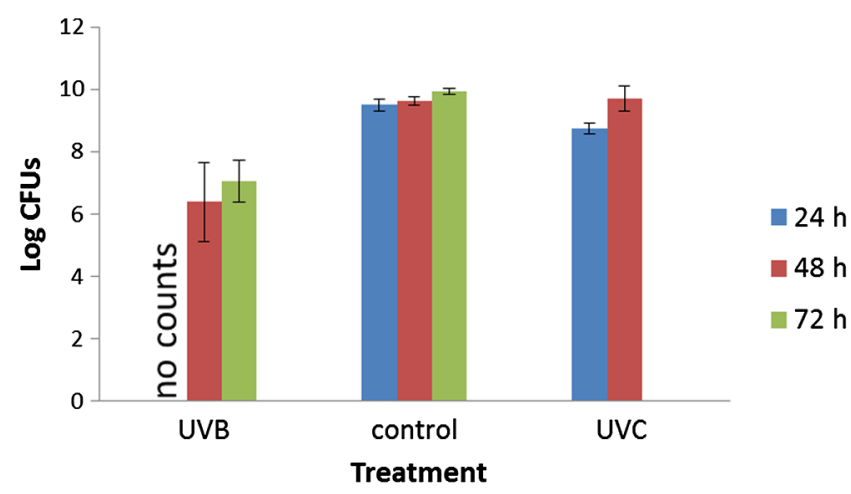

Fig. $6 \mathrm{CFU}$ regrowth after different treatments are applied (UVB 20,000 , UVC $20,000 \mathrm{~J} / \mathrm{m}^{2}$, and control) on biofilms at different maturity stages $(24,48$, and $72 \mathrm{~h}$ grown). The error bars represent the standard deviation. No counts were observed for all replicates after the UVB treatment was applied on $24 \mathrm{~h}$ grown biofilms. The UVC treatment was not performed on the $72 \mathrm{~h}$ grown biofilms since only modest inactivation was observed for biofilms grown for $48 \mathrm{~h}$ (missing green column for the UVC treatment). CFUs are per initial volume.

The UVB treatment was equally efficient for killing 48 and $72 \mathrm{~h}$ mature biofilms $(p=0.6)$.

\subsection{Transmission Properties of Biofilms in the Ultraviolet Region}

Transmission properties of biofilms in the UV region were measured with a Cary 50 spectrophotometer. The membrane filters on which the original biofilms were grown were opaque in the UV region. Therefore, biofilms were grown on (UV transparent) quartz surfaces (three samples). To grow these biofilms, quartz surfaces were embedded in bacterial culture for $28 \mathrm{~h}$. It cannot be expected that a biofilm grown on quartz will attain the same structure, thickness, and properties as a biofilm grown on a membrane filter. Moreover, the biofilm thickness is not expected to be the same over the whole quartz surface, confirmed by observation of the samples by bare eye. The thickness variation of the biofilm grown at different locations on one quartz plate ("biofilm spot") is attributed to the biological aspects of biofilm formation. Therefore, large variation for the absolute values of the transmission was expected; the location on the biofilm where the transmission measurement was taking place was not controllable. 


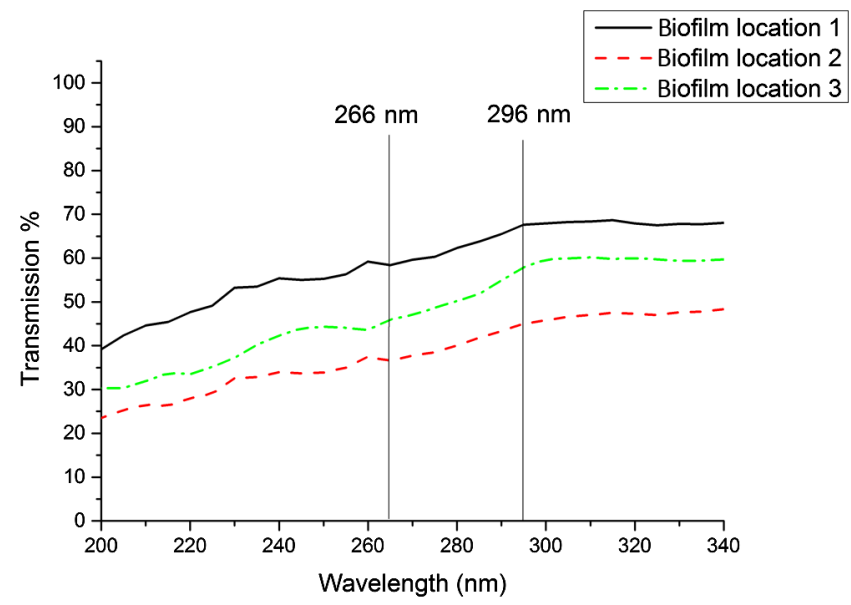

Fig. 7 Transmission spectra of biofilms grown on quartz under the exact same conditions. It is observed that on average $10 \%$ more UV light is transmitted at a wavelength of $296 \mathrm{~nm}$ than at $266 \mathrm{~nm}$. Only $37 \%$ is transmitted at $266 \mathrm{~nm}$ for biofilm location 2 .

The transmission properties of biofilms grown on quartz (Fig. 7) and, more specifically, the ratio of transmission properties at different wavelengths could assist in obtaining a better understanding of the UV wavelength-dependent penetration ability into a thin layer of bacteria existing in a "biofilm state."

It was observed that on average (three different spots, each on a different biofilm quartz sample, were measured) $10 \%$ less UV light was transmitted at a wavelength of $266 \mathrm{~nm}$ than at $296 \mathrm{~nm}$, the transmission curve slope was found to be $0.33 \pm 0.07$. In the case of biofilm location 2 (obviously the thickest among the "biofilm spots" measured), only $37 \%$ was transmitted at a wavelength of $266 \mathrm{~nm}$ (Fig. 7).

Therefore, it is believed that, in the case of the biofilms grown on filters, which were much thicker, the UVC irradiation did not penetrate through the whole biofilm volume.

\section{Discussion}

To achieve no regrowth after treatment with UV irradiation, it is assumed that the penetration depth of the UV wavelength should be such that the dose arriving at the deepest layer of biofilm equals the lethal dose 100 (LD100, defined as the dose resulting in $100 \%$ inactivation). It is expected that the effectiveness of the UV treatment will be strongly correlated to the thickness as well as the opaqueness of the biofilm. If for some reason (e.g., increased thickness) the delivered dose at the deepest layer is reduced, it is expected that the growth observed can be increased by several orders of magnitude, due to bacterial survival. The typical thickness for both less mature and mature biofilms is $\sim 200 \mu \mathrm{m}$ or more. It is probable that the thickness and opaqueness of the biofilms vary significantly among different ON cultures and even among technical replicates. Therefore, variance of the inactivation effect, achieved by UVB-effective treatments, should be expected to be observed, depending on the biofilm properties. This was confirmed by the experimental process (Figs. 3-5). For ineffective UV treatments, the variance should be masked since insignificant inactivation effects are induced in all replicates. To our knowledge, the ability to eliminate a biofilm with UVB LEDs (narrowband spectral power distribution) more effectively than UVC has not previously been reported. If in the future, UV treatments are used for clinical purposes should not exceed 15 min for convenience. All treatments in the present paper did not exceed $12 \mathrm{~min}$, despite the power constraints existing at the moment for AlGaN UV LEDs. ${ }^{29}$ The term inactivation is used in a broader context that includes both possibilities of either killing the biofilm or inactivating it.

Modeling of the radiant exposure dependent killing of the $P$. aeruginosa biofilms with UVC and UVB light showed that a log-linear model (based on first-order kinetics) failed to fit the experimental data (in the tested range for radiant exposure, namely from 70 to $10,000 \mathrm{~J} / \mathrm{m}^{2}$ ) and failed to provide a satisfactory estimate for the required radiant exposure for achieving a 4-log CFU reduction. On the contrary, more complex models that take into account a "shift of behavior" (Weibull, biphasic, $\log +$ tail, Albert) for a subgroup of bacteria succeeded in fitting the experimental data $\left(R^{2} \geq 0.9\right)$. Moreover, the "Weibull model" was adequate for giving a rough estimation/prediction (correct order of magnitude) of the reliable dose $d_{R}$, for both UVB and UVC treatments. Weibull models have been reported to successfully predict inactivation of Escherichia coli and Salmonella enterica after exposure to ozone or pulsed light (100 to $1100 \mathrm{~nm}) .^{30}$ Finally, $\beta$ values were $<1$ for both UVB and UVC treatments, meaning that there are some persistent bacteria in the biofilm that have less probability of being killed, perhaps because they adapt to the irradiation or because they are not as affected, e.g., due to limited penetration depth of light to the biofilm's deepest layers and/or due to the shielding effect of the extracellular polymeric substances.

\section{Conclusion and Outlook}

Different UV treatments were applied on $P$. aeruginosa biofilms, namely UVB irradiation and UVC irradiation, and the effect of the treatments on the ability of the biofilm to regrow was studied quantitatively.

For biofilms grown for $24 \mathrm{~h}$, it was shown that the biofilm survival could not be described and predicted sufficiently by first-order kinetics (in the tested range for radiant exposure, namely from 70 to $10,000 \mathrm{~J} / \mathrm{m}^{2}$ ). The "Weibull model" was successful in giving a prediction of the reliable dose $d_{R}$, for the UVB treatment. From the survival curves (tested in the range from 70 to $10,000 \mathrm{~J} / \mathrm{m}^{2}$ ), it was concluded that UVB irradiation is much more efficient in inactivating $P$. aeruginosa biofilm (24 $\mathrm{h}$ grown) than UVC.

Moreover, it was demonstrated that the efficacy of the UVB treatment $\left(20,000 \mathrm{~J} / \mathrm{m}^{2}\right)$ drops drastically when performed on more mature biofilms. While total inactivation was observed for $24 \mathrm{~h}$ grown biofilms, only a 3-log reduction was achieved (on average) on mature biofilms (48 and $72 \mathrm{~h}$ grown). The UVB treatment was equally efficient for killing 48 and $72 \mathrm{~h}$ mature biofilms $(p=0.6)$. Furthermore, the success of the UVB treatment varied when the biofilms were mature, but it was "straightforward" for less mature biofilms (no counts observed in all cases). The UVC treatment $\left(20,000 \mathrm{~J} / \mathrm{m}^{2}\right)$ could not be considered a successful treatment independently of the maturity stage of the biofilm; inactivation did not exceed $1-\log$ even at $20,000 \mathrm{~J} / \mathrm{m}^{2}$. Nontreated samples (control) exhibited a slight increase in counts with maturity $(p=0.003)$. The UVB treatment $\left(20,000 \mathrm{~J} / \mathrm{m}^{2}\right)$ was significantly more effective than UVC $\left(20,000 \mathrm{~J} / \mathrm{m}^{2}\right)$, both for biofilms grown for 24 and $48 \mathrm{~h}(p \leq 0.004)$.

Since the effectiveness of treatments involving UV irradiation clearly depends on the thickness/opaqueness of the biofilm, it is crucial to investigate in the future the correlations among the variables: biofilm thickness, biofilm opaqueness for various UV wavelengths, biofilm maturity, species of bacteria present in the 
biofilm, and inactivation efficiency of UV wavelengths. The fact that UVB irradiation (wavelength of $296 \mathrm{~nm}$ ), which exists in daylight, has such disinfection ability on biofilms reveals new aspects for direct exposure to sunlight and frames some prospect for indoor illumination using UV-transparent windows. The biofilm inactivation ability of LEDs in the UVB range offers new possibilities for applications not only in terms of disinfecting instruments but also for treatment of infectious diseases.

\section{Disclosures}

The authors declare that there is no conflict of interest.

\section{Acknowledgments}

The authors would like to thank Anne Nielsen for her technical assistance in the laboratory and Sabrina Gericke for the blind counting of the CFUs. Moreover, the authors would like to acknowledge that the present publication is a derivation of prior SPIE Photonics Europe Conference Proceedings. ${ }^{31}$

\section{References}

1. J. P. Guggenbichler et al., "Incidence and clinical implication of nosocomial infections associated with implantable biomaterials-catheters, ventilator-associated pneumonia, urinary tract infections," GMS Krankenhaushygiene Interdisziplinär 6(1), 1-19 (2011).

2. T. Bjarnsholt, "The role of bacterial biofilms in chronic infections," APMIS 121(136), 1-58 (2013).

3. J. D. Bryers, "Medical biofilms," Biotechnol. Bioeng. 100(1), 1-18 (2008).

4. A. Geddes, "Infection in the twenty-first century: predictions and postulates," J. Antimicrob. Chemother. 46(6), 873-877 (2000).

5. A. Van Leeuwenhoek, "Microscopical observations about animals in the scurf of the teeth," Philos. Trans. R. Soc. Lond. B Biol. Sci. 14, 568-574 (1684).

6. S. A. Jassim and R. G. Limoges, "Natural solution to antibiotic resistance: bacteriophages 'The Living Drugs'," World J. Microbiol. Biotechnol. 30(8), 2153-2170 (2014).

7. P. D. Cotter, R. P. Ross, and C. Hill, "Bacteriocins-a viable alternative to antibiotics?" Nat. Rev. Microbiol. 11(2), 95-105 (2013).

8. R. Yin et al., "Light based anti-infectives: ultraviolet C irradiation, photodynamic therapy, blue light, and beyond," Curr. Opin. Pharmacol. 13(5), 731-762 (2013).

9. T. P. Thai et al., "Ultraviolet light $\mathrm{C}$ in the treatment of chronic wounds with MRSA: a case study," Ostomy/Wound Manage. 48(11), 52-60 (2002).

10. T. P. Thai et al., "Effect of ultraviolet light $\mathrm{C}$ on bacterial colonization in chronic wounds," Ostomy/Wound Manage. 51(10), 32-45 (2005).

11. M. R. Hamblin and T. Hasan, "Photodynamic therapy: a new antimicrobial approach to infectious disease?" Photochem. Photobiol. Sci. 3(5), 436-450 (2004).

12. T. Maisch et al., "Combination of $10 \%$ EDTA, photosan, and a blue light hand-held photopolymerizer to inactivate leading oral bacteria in dentistry in vitro," J. Appl. Microbiol. 107(5), 1569-1578 (2009).

13. C. S. Enwemeka et al., "Blue 470-nm light kills methicillin-resistant staphylococcus aureus (MRSA) in vitro," Photomed. Laser Surg. 27(2), 221-226 (2009).

14. T. Dai et al., "Blue light rescues mice from potentially fatal Pseudomonas aeruginosa burn infection: efficacy, safety, and mechanism of action," Antimicrob. Agents Chemother. 57(3), 1238-1245 (2013).

15. K. T. Tsen et al., "Photonic approach to the selective inactivation of viruses with a near-infrared subpicosecond fiber laser," J. Biomed. Opt. 14, 064042 (2009).

16. K. T. Tsen et al., "Selective inactivation of micro-organisms with nearinfrared femtosecond laser pulses," J. Phys. Condens. Matter 19(47), 472201 (2007).

17. A. H. Geeraerd, V. P. Valdramidis, and J. F. Van Impe, "GInaFiT, a freeware tool to assess non-log-linear microbial survivor curves," Int. J. Food Microbiol. 102(1), 95-105 (2005).
18. L. L. Barnkob et al., "Investigation of the effect of UV-LED exposure conditions on the production of vitamin D in pig skin," Food Chem. 212, 386-391 (2016).

19. C. K. Stover et al., "Complete genome sequence of Pseudomonas aeruginosa PAO1, an opportunistic pathogen," Nature 406(6799), 959-964 (2000).

20. T. Bjarnsholt et al., "Antibiofilm properties of acetic acid," Adv. Wound Care 4(7), 363-372 (2015).

21. M. A. Hamilton and A. E. Parker, "Enumerating viable cells by pooling counts for several dilutions," Testing Surface Disinfectants, a project of the Standarized Biofilm Methods Laboratory in the CBE, http://www. biofilm.montana.edu/documents/KSA-SM-06.pdf (22 September 2010).

22. W. D. Bigelow and J. R. Esty, "The thermal death point in relation to typical thermophylic organisms," J. Infect. Dis. 27, 602-617 (1920).

23. A. H. Geeraerd, C. H. Herremans, and J. F. Van Impe, "Structural model requirements to describe microbial inactivation during a mild heat treatment," Int. J. Food Microbiol. 59(3), 185-209 (2000).

24. P. Mafart et al., "On calculating sterility in thermal preservation methods: application of the Weibull frequency distribution model," Int. J. Food Microbiol. 72, 107-113 (2002).

25. I. Albert and P. Mafart, "A modified Weibull model for bacterial inactivation," Int. J. Food Microbiol. 100, 197-211 (2005).

26. O. Cerf, "Tailing of survival curves of bacterial spores," J. Appl. Bacteriol. 42, 1-19 (1977).

27. M. A. J. S. van Boekel, "On the use of the Weibull model to describe thermal inactivation of microbial vegetative cells," Int. J. Food Microbiol. 74, 139-159 (2002).

28. H. Naghili et al., "Validation of drop plate technique for bacterial enumeration by parametric and nonparametric tests," Vet. Res. Forum 4(3), 179 (2013).

29. T. Nishida, H. Saito, and N. Kobayashi, "Efficient and high-power AlGaN-based ultraviolet light-emitting diode grown on bulk GaN," Appl. Phys. Lett. 79(6), 711-712. (2001).

30. K. L. Bialka, A. Demirci, and V. M. Puri, "Modeling the inactivation of Escherichia coli O157: H7 and Salmonella enterica on raspberries and strawberries resulting from exposure to ozone or pulsed UV-light," Int. J. Food Eng. 85(3), 444-449 (2008).

31. A. Argyraki et al., "Comparison of UVB and UVC irradiation disinfection efficacies on Pseudomonas Aeruginosa (P. aeruginosa) biofilm," Proc. SPIE 9887, 988730 (2016).

Aikaterini Argyraki has a master of science in physics and nanotechnology and is currently working as a PhD student on the development of new light sources for biomedical applications at the Department of Photonics Engineering, DTU.

Merete Markvart has several peer-reviewed publications. Her research focuses on prevention and treatment of endodontic infection focusing on preparation techniques, root morphology, and antibacterial treatment procedures.

Lars Bjørndal is a recognized worldwide expert within clinical handling of biofilm-associated diseases on dental tissues, in particularly caries and apical periodontitis. He has numerous peer-reviewed articles and book chapters involving this field. He participated recently in an International Consensus Group covering terminology and handling of caries. His research topics involve quality shaping factors within endodontics and the comparison and long-term evaluation of various dental treatments using randomized clinical trials.

Thomas Bjarnsholt is a global expert in biofilms, with over 135 peer-reviewed publications in the biofilm field, including the recent ESCMID guidelines for biofilm treatment. His research focuses on biofilm formation and the role of bacterial biofilm in chronic infections, both in vitro, animal models and ex vivo material from chronic infections.

Paul Michael Petersen is a full professor in new light sources at the Technical University of Denmark. He has more than 20 years of research experience in lasers, LEDs, and optical measuring techniques. From 2002 until 2012, he was appointed adjunct professor in optics at the Niels Bohr Institute, Copenhagen University. He has authored more than 170 international scientific publications and holds 15 patents. 